\title{
Análisis del uso del póster científico y de la revisión por pares como herramienta desarrollo de la competencia comunicación efectiva en estudiantes de grado en ingeniería
}

\section{Gema Prats-Boluda ${ }^{\mathrm{a}}$, Yiyao Ye Lin ${ }^{\mathrm{a}}$ y Beatriz Ana Trénor Gomis ${ }^{\mathrm{a}}$}

a Universitat Politècnica de València. Departamento de Ingeniería Electrónica (geprabo@eln.upv.es; yiye@eln.upv.es; btrenor@eln.upv.es).

\begin{abstract}
Today's society requires graduates who present a wide range of cross skills in addition to master the specific skills of their grade, as in the case of effective communication skill. In this paper the use of scientific-technical poster as a communication tool for undergraduate engineering students is studied, analyzing whether peer review teaching strategy can improve the students' writing-visual communication skills. Additionally, the burden associated with the use of this strategy and the students' degree of acceptance is studied. Experimental results indicate that both students and teachers have positively assessed the use of the scientific-technical poster as an effective tool for developing the students' communication skills. Furthermore, peer review process has identified numerous common errors in the posters made by the students, improving their skill development without excessive additional burden. 92.3\% of the surveyed students consider that peer review is a good teaching strategy for developing communication skills and it should be extended to other subjects and/or courses.
\end{abstract}

Keywords: Peer review, effective communication skills, cross skills

\begin{abstract}
Resumen
La sociedad actual requiere de egresados que además de dominar las competencias específicas de su título presenten un amplio abanico de competencias transversales como es el caso de la comunicación efectiva. En el presente trabajo se valora el uso del póster científico-técnico como herramienta de comunicación para los alumnos de grado en ingeniería, analizando si el empleo de la revisión por pares permite a los estudiantes mejorar esta competencia. Asimismo se valora el grado de aceptación de esta metodología por parte del alumnado y si supone una carga adicional excesiva o no. Los resultados de la experiencia indican que tanto el alumnado como el profesorado valoran positivamente el desarrollo y defensa de un póster científico-técnico como herramienta de trabajo de la competencia comunicación efectiva. Además se evidencia que el proceso de la revisión por pares ha permitido identificar un gran número de errores presentes en los pósteres elaborados por los alumnos, ayudándoles a una mejor adhesión de la competencia, sin suponer una carga adicional excesiva.
\end{abstract}


Análisis del uso del póster científico y de la revisión por pares como herramienta desarrollo de la competencia comunicación efectiva en estudiantes de grado en ingeniería

El 92.3\% de los alumnos encuestados de la asignatura opinan que es una buena herramienta docente que debería emplearse en otras asignaturas y/o cursos.

Palabras claves: revisión por pares, comunicación efectiva, competencias transversales,

\section{Introducción}

La adaptación y asimilación del Espacio Europeo de Educación Superior (EEES) ha supuesto una sustancial transformación de la docencia universitaria, tanto en lo referente a las metodologías empleadas, en las estructuras de las enseñanzas, o en la implantación de sistemas que regulación y control de su calidad (Hermosilla 2013). En la actualidad el foco ha dejado de centrarse sólo en las competencias específicas de cada titulación y ha revelado la necesidad de formar en competencias transversales, que permitan a los egresados adquirir de forma continua las habilidades y destrezas demanda la actual sociedad del conocimiento.

Las competencias genéricas o transversales se pueden clasificar en: Instrumentales (análisis y síntesis, aprendizaje autónomo, organización y planificación, resolución de problemas, toma de decisiones, comunicación oral y escrita y conocimientos de lenguas extranjeras), Competencias Interpersonales (trabajo en equipos multidisciplinares, reconocimiento de la diversidad y multiculturalidad, razonamiento crítico, compromiso ético, negociación y auto-motivación) y Sistémicas (creatividad, liderazgo, iniciativa y espíritu emprendedor, adaptación a nuevas situaciones, preocupación por la calidad, sensibilidad frente a temas medioambientales, gestión de proyectos y gestión por objetivos) (Marquez 2011).

La comunicación efectiva se enmarca dentro del grupo de las competencias transversales instrumentales e implica utilizar los recursos adecuados para poder transmitir información, ideas, problemas y soluciones adaptándose al contexto y al receptor para conseguir su correcta comprensión (Solano 2011). Esta competencia se sitúa en la parte superior de la lista de lo que los empresarios buscan en los graduados.

Generalmente se asocia a la comunicación oral y escrita, aunque cada día cobra más fuerza el aspecto visual. Así, el póster científico/técnico es una herramienta ampliamente empleada en el ámbito de la ingeniería, tanto en lo relativo al mundo industrial (ferias, exposiciones) como a nivel de investigación y docencia. Pese a ello los alumnos reciben poca formación a este respecto a lo largo de su trayectoria curricular. Por esta razón en el presente trabajo se presenta la experiencia llevada a cabo en la asignatura Bioelectrónica (optativa de cuarto curso del grado de ingeniería electrónica industrial y automática, ETSID UPV) en la que los alumnos deberán preparar y defender un póster científico/técnico sobre diferentes temas de la asignatura.

Existen diversas metodologías docentes para ayudar a los alumnos a desarrollar la competencia comunicación escrita o visual. La metodología revisión por pares es ampliamente utilizada en el ámbito de investigación (Guiford 2001) y también en la formación de los estudiantes de ingeniería y medicina (Moon Sook 2011; Riemer 2007; Stephanie 2000). La revisión de pares consiste en evaluar el trabajo borrador de un compañero con similar nivel de conocimientos y de experiencia, y proporcionar la

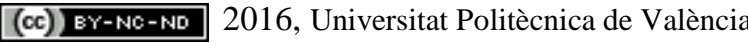


retroalimentación constructiva sobre la comprensibilidad, estructura, lógica y flujo, ajuste a los criterios de evaluación. De esta manera los alumnos tienen oportunidad para mejorar los borradores antes de que sea demasiado tarde. La revisión por pares permite incrementar la motivación de aprendizaje además de fomentar el pensamiento crítico de los alumnos. Esta técnica también mejora el nivel de confianza en sí mismo después de haber evaluado por otros en un entorno profesional. Pese a los numerosos beneficios mencionados anteriormente, esta metodología no está exenta de algunos inconvenientes para su implantación en entornos universitarios, entre ellos se puede citar: requiere mayor tiempo para realizar el trabajo lo cual podría suponer una carga adicional excesiva para el alumno, algunos estudiantes creen que no necesitan mejorar su escritura y muestran actitud negativa a la metodología. Por consiguiente en este trabajo se valorará la implantación de la metodología de revisión por pares para mejorar la competencia comunicación efectiva. .

\section{Objetivos}

El presente trabajo aborda un doble objetivo. En primer lugar pretende trabajar la competencia comunicación efectiva en la asignatura Bioelectrónica (optativa grado en Ingeniería Electrónica Industrial y Automática, ETSID) mediante la elaboración y defensa de un póster científico, analizando a su vez si la revisión por pares permite mejorar dicha competencia en los estudiantes. Asimismo se valorará el grado de aceptación de esta metodología por parte del alumnado y si la perciben como una carga adicional excesiva o no.

\section{Desarrollo de la innovación}

La experiencia que se presenta en el presente trabajo se desarrolla en el contexto de la asignatura optativa Bioelectrónica ofertada en cuarto curso de la titulación Grado en Ingeniería Electrónica Industrial y Automática, cuatrimestre B. Se trata de una asignatura con un amplio temario que abarca desde la descripción y origen electrofisiológico y mecánico de las principales señales biomédicas empleadas en clínica y en investigación, sus sistemas de captación, instrumentación, aplicabilidad diagnóstica y últimos avances al respecto a nivel de investigación. Se trata de un temario del que los alumnos o bien tienen conocimientos básicos lejanos (secundaria) o ninguno, p.e. electrofisiología en general. Con el objetivo de dinamizar dicha asignatura, aumentando el grado de motivación de los alumnos, así como de trabajar diferentes competencias transversales además de las específicas, en los últimos años se han incluido diferentes metodologías de enseñanza aprendizaje y técnicas de evaluación en la misma. Señalar que la experiencia que se expone se ha llevado a cabo en el curso académico 15-16. Concretamente, el sistema de evaluación de la asignatura en dicho curso consiste en: 2 trabajos teóricos. Uno de ellos presentado mediante un póster -y sobre el que versa la presente experiencia- (15\%) y el otro en una presentación oral y memoria escrita (20\%). Además los alumnos realizan 2 prácticas de laboratorio, entregando sendas memorias al final de las sesiones (20\%). Finalmente los alumnos deberán realizar un trabajo práctico en grupo sobre un sistema de adquisición de señales biomédicas (electrocardiógrafo, neumógrafo) que será evaluado mediante la entrega de un diario de laboratorio (30\%) y la demostración experimental del funcionamiento de

(cc) BY-Nc-ND 2016, Universitat Politècnica de València

Congreso In-Red (2016) 
dicho sistema (15\%). Todas estas actividades son voluntarias. Destacar además que la asignatura presenta 18 alumnos matriculados en el curso 15-16.

En cuanto a la planificación docente relativa al trabajo teórico expuesto mediante póster, ésta consiste en una sesión inicial de 4.5h. En ella se forman, de forma libre, grupos de tres alumnos para su realización. Los temas a desarrollar (ver Anexo I) son expuestos por la profesora y se deja a los diferentes grupos la selección del tema que desean desarrollar. En caso de que varios grupos quieran llevar a cabo el mismo tema se asigna por sorteo en el mismo momento delante de todos los alumnos. Este proceso de formación y asignación de trabajos conlleva aproximadamente unos 20 minutos. Una vez asignados los trabajos la profesora explica a los alumnos cómo hacer búsquedas en repositorios y bases de datos científicos (web of science, google scholar). Asimismo en dicha sesión la profesora proporciona parte del material bibliográfico y supervisa y orienta las búsquedas por internet. Concretamente la profesora responsable de la actividad les cuelga en la web de la asignatura un artículo de referencia para la elaboración del póster (Guardiola 2010), así como un ejemplo de un buen póster técnico. Además durante la sesión inicial se les facilita bibliografía de referencia -libros y artículos científicos indexados JCR- para los temas propuestos.

Tras esta primera sesión los alumnos disponen de 2 semanas para la elaboración de la primera versión del póster (tamaño A1). Transcurrido este periodo lo envían a la profesora responsable de la actividad. El póster no debe contener el nombre de los alumnos que lo han elaborado, de manera que esta información sólo la conoce la profesora. Ésta se encarga de reenviar esta primera versión a los diferentes grupos para que hagan una revisión por pares ciega. Los alumnos utilizan cómo rúbrica de la corrección las directrices expuestas en el artículo de Guardiola (Guardiola 2010). Los alumnos disponen de una semana para llevar a cabo la revisión y enviar a la profesora sus comentarios. Ésta los supervisa y los reenvía a los grupos correspondientes. A partir de este momento los alumnos tienen otra semana para hacer los cambios/modificaciones pertinentes y preparar la exposición oral (7 minutos por grupo). Finalmente se dedica una sesión de $2 \mathrm{~h}$ para la exposición de los pósteres que se desarrolla en el Hall de la ETSID (previa reserva anticipada del espacio por parte del profesorado puesto que hay que determinar el número de parabanes necesarios y su ubicación). Indicar que la impresión de los pósteres no ha supuesto un coste al alumnado puesto que han sido impresos empleando los recursos de la ETSID con el permiso pertinente. Al finalizar la sesión los alumnos cumplimentan el cuestionario que se adjunta ene l Anexo II de este documento.

\section{Resultados}

De los 18 alumnos matriculados en la asignatura, se formaron inicialmente 7 grupos de 2 o 3 alumnos. De ellos, no hubo seguimiento del trabajo de un grupo de 3 alumnos, es decir, no realizaron ni presentaron el trabajo asignado por motivos diversos. Esto probablemente se debe a que la actividad tiene un carácter voluntario y tiene una ponderación del 15\% de la nota final de la asignatura. Además hubo un grupo formado por 2 alumnos por motivos diversos realizaron el trabajo pero ausentaron en la sesión de la presentación del póster. Por lo que la encuesta se realizó a un total de 13 alumnos.

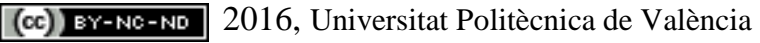


Igual que los cursos anteriores las profesoras de la asignatura han percibido una participación activa en la clase de los estudiantes para la búsqueda de información en la bibliografía proporcionado por parte del profesor y en los recursos internet. De la primera versión del póster elaborado por los alumnos (antes de enviarlos a los alumnos para su revisión), las profesoras de la asignatura hicieron una valoración cualitativa y encontraron que la calidad de los pósteres es bastante similar a los de cursos anteriores.

En la tabla 1 se adjuntan los comentarios y/o sugerencias que recibieron cada trabajo. Las faltas comunes en cuestión del formato del póster que han identificado los revisores son "No incluye pie de figura"; "No incluyen Referencias Bibliográficas en el texto"; "calidad de imagen inadecuada"; "No resalta la información relevante del trabajo o formato inadecuado para ello"; "No indica el significado de las abreviaturas". Estos resultados coinciden con las observaciones de las profesoras de la asignatura en los pósteres elaborados por alumnos de cursos anteriores. Respecto al contenido del trabajo, los revisores han sugerido que incluyen cierta información específica o una descripción más detalle de algún contenido, todo ello con el objetivo de ayudar al lector para seguir el hilo de explicación sobre el trabajo. Las profesoras consideraron que la inclusión de la información complementaria sugerida por los revisores y la corrección de las faltas comunes en el formato ha permitido una mejora considerable en la calidad del póster tras la revisión por pares.

La figura 1 muestra los resultados del cuestionario adjunto en el Anexo II, siendo el total de alumnos encuestados 13. En general los alumnos opinan que los ponentes dominan la materia del trabajo asignado. La distribución de los trabajos más atractivos es bastante equitativa, siendo los trabajos no 6 (30.8\%) y 5 (23.1\%) son más atractivos. En cambio, los alumnos consideran que el trabajo $\mathrm{n}^{\circ} 2$ se ha explicado con mayor claridad.

La mayoría de los grupos han invertido entre 10 y 20 h (69.2\%) para recopilar la información necesaria y entre 5-15 h (61.5\%) para la elaboración de la primera versión del póster. En cuanto al tiempo requerido para la revisión de los pósteres de los compañeros se reparte aproximadamente entre menos de una hora (38.5\%) y entre 1-3 h (68.5\%). Del mismo modo el tiempo invertido para llevar a cabo las modificaciones conforme a los comentarios de los revisores se reparte casi por igual entre menos de una hora (46.2\%) y entre 1-5 h (53.8\%). Por tanto, el tiempo medio invertido por grupo para elaborar la primera versión del póster oscila entre 15-35 h, y el tiempo total teniendo en cuenta el tiempo adicional para la revisión por pares asciende a 17-43 h. Estos resultados sugieren que la revisión por pares no supone una excesiva carga adicional para los alumnos en comparación con el tiempo necesario para la elaboración de la primera versión del póster.

La mayoría de los alumnos consideran que los comentarios de revisores eran objetivos y les ha ayudado a mejorar el contenido del trabajo a exponer. El 92.3\% de los alumnos encuestados consideran que la revisión por pares es una buena metodología docente y están a favor de su implantación en cursos posteriores.

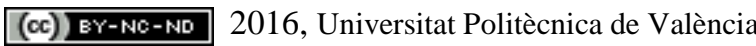

Congreso In-Red (2016) 
Análisis del uso del póster científico y de la revisión por pares como herramienta desarrollo de la competencia comunicación efectiva en estudiantes de grado en ingeniería

Tabla 1. Comentarios/sugerencias de los revisores: algunos relacionados con el formato del póster y otros sobre el contenido del mismo.

\begin{tabular}{|c|c|c|}
\hline Trabajo & Formato & Contenido \\
\hline 1 & $\begin{array}{l}\text { F11 Tamaño del título demasiado pequeño } \\
\text { F12 No hay conclusiones } \\
\text { F13 Subrayado en los registros de respiración } \\
\text { no aconsejable } \\
\text { F14 Títulos de los distintos apartados no } \\
\text { resaltan lo suficiente } \\
\text { F15 No incluye pie de figuras }\end{array}$ & $\begin{array}{l}\text { C11 Expresión incorrecta en "El sistema } \\
\text { respiratorio es el proceso encargado de } \\
\text { aportar..." El sistema respiratorio no es un } \\
\text { proceso, sino una estructura anatómica. } \\
\text { C12 Imprecisión en la descripción de las } \\
\text { funciones de respiración “Transportar } \mathrm{O}_{2} \text { y } \mathrm{CO}_{2} \\
\text { por la sangre” dado que el sistema respiratorio no } \\
\text { es en sí el encargado de transportar gases por la } \\
\text { sangre, sino intercambiarlos con el exterior. } \\
\text { C13 No incluye parámetros característicos de la } \\
\text { señal de respiración registrada con los } \\
\text { transductores }\end{array}$ \\
\hline 2 & $\begin{array}{l}\text { F21 No indica el significado de las } \\
\text { abreviaturas. } \\
\text { F22 No incluyen "Referencias } \\
\text { Bibliográficas" }\end{array}$ & $\begin{array}{l}\text { C21 No queda claro si los músculos esqueléticos, } \\
\text { el músculo liso y las glándulas son efectores } \\
\text { C22 Imprecisión "Estándar 10-20 para EEG" y } \\
\text { debería ser "Sistema estándar 10-20 para la } \\
\text { adquisición de EEG" }\end{array}$ \\
\hline 3 & $\begin{array}{l}\text { F31 Demasiado texto y pocas figuras } \\
\text { F32 Figura } 5 \text { faltan los valores del eje de } \\
\text { ordenadas. La leyenda de la gráfica no } \\
\text { coinice con el pie de figura } \\
\text { F33 Calidad de imagen inadecuada } \\
\text { F34 No indica en el texto las "Referencias } \\
\text { bibliográficas" }\end{array}$ & $\begin{array}{l}\text { C31 Describa con detalle el "Tipo de electrodos } \\
\text { para la captación de las señales } \\
\text { electromiográficas y la colocación de los } \\
\text { mismos" }\end{array}$ \\
\hline 4 & $\begin{array}{l}\text { F41 A excepción del título de los apartados, } \\
\text { no se resalta la información relevante } \\
\text { F42 Hay figuras sin pie de figura } \\
\text { F43 Calidad de imagen (intestino delgado) } \\
\text { inadecuada }\end{array}$ & $\begin{array}{l}\text { C41 No incluye la amplitud de la señal eléctrica } \\
\text { del colon }\end{array}$ \\
\hline 5 & $\begin{array}{l}\text { F51 Falta de ortografía } \\
\text { F52 El apartado "Señal mioeléctrica uterina” } \\
\text { utiliza tamaño de texto demasiado pequeño y } \\
\text { muy denso } \\
\text { F53 Línea horizontal a mitad del póster } \\
\text { innecesaria }\end{array}$ & C51 Redacción de conclusión mejorable \\
\hline 6 & $\begin{array}{l}\text { F61 Falta el apartado de objetivos } \\
\text { F62 Bibliografía demasiado extenso } \\
\text { F63 Tamaño de letra inadecuado y no } \\
\text { homogéneo } \\
\text { F64 No incluyen "Referencias bibliográficas” } \\
\text { en el texto } \\
\text { F65 Mala calidad de imagen. }\end{array}$ & $\begin{array}{l}\text { C61 Descripción pobre de la señal de marcha } \\
\text { C62 No presenta el análisis de los datos de } \\
\text { tomografía por emisión de positrones } \\
\text { C63 Debe incluir la instrumentación de la señal } \\
\text { de marcha y el análisis de datos } \\
\text { C64 Imprecisión en "Freezing of gate" cuando se } \\
\text { trata "Freezing of gait" (sin explicación) } \\
\text { C65 Mayor hincapié en el apartado de } \\
\text { tratamientos }\end{array}$ \\
\hline
\end{tabular}




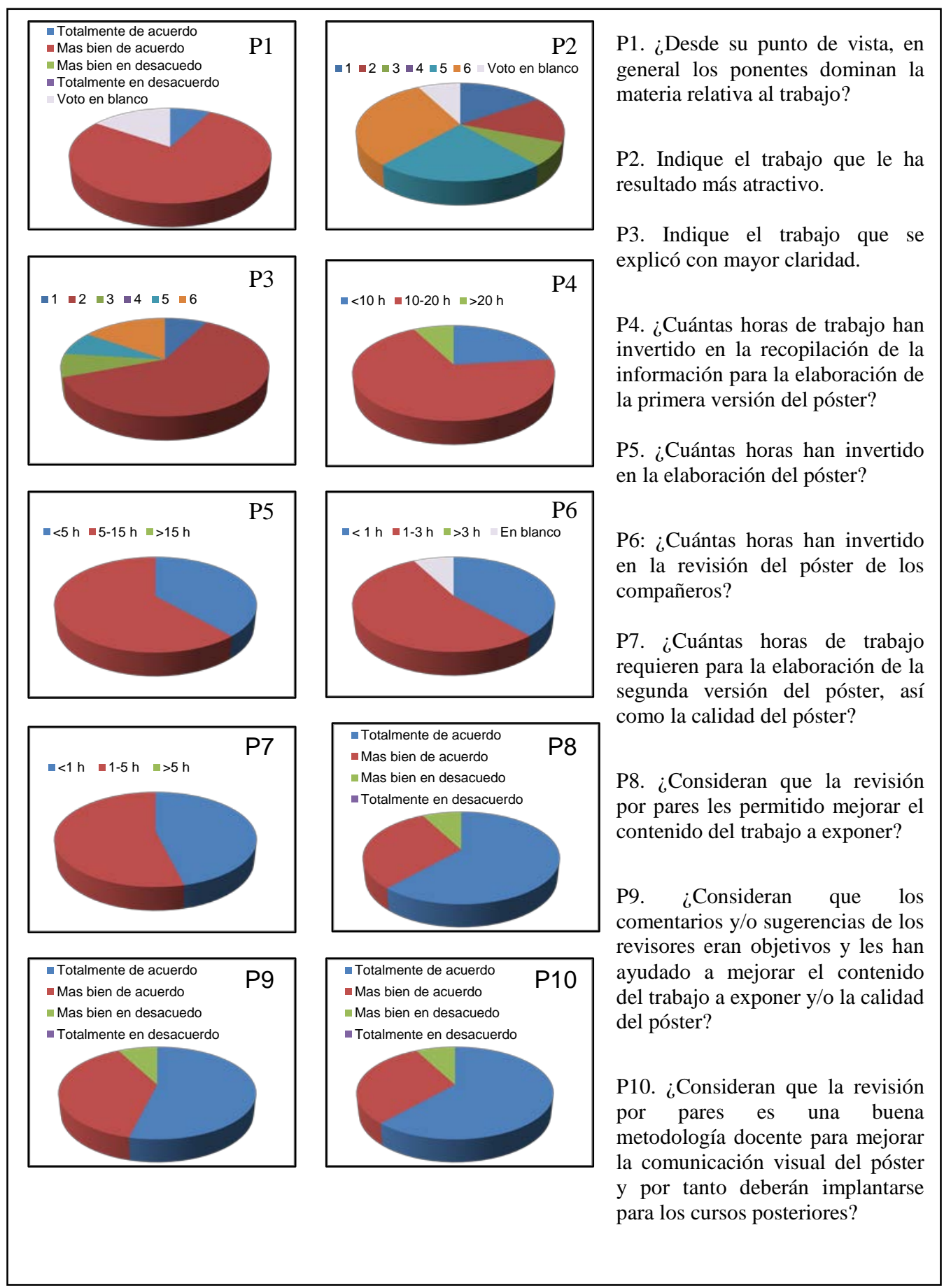

Fig. 1 A la derecha se detallan las preguntas de la encuesta realizada a los alumnos ( $n^{\circ}$ total de alumnos encuestados 13). A la izquierda se representan los resultados de cada una de las preguntas. 
Análisis del uso del póster científico y de la revisión por pares como herramienta desarrollo de la competencia comunicación efectiva en estudiantes de grado en ingeniería

\section{Conclusiones}

Los alumnos y el profesorado valoran positivamente el desarrollo y defensa de un póster científico-técnico como herramienta de trabajo de la competencia transversal comunicación efectiva. Asimismo la revisión por pares ha permitido identificar un gran número de faltas comunes presentes en los documentos escritos elaborados por los alumnos, y por tanto permite a los estudiantes mejorar dicha competencia sin suponer una carga adicional excesiva. El 92.3\% de los alumnos encuestados de la asignatura opinan que es una buena metodología docente que debería implantarse en los cursos posteriores.

\section{Referencias}

GUARDIOLA E. (2010) El póster científico. Cuaderno de la Fundación Dr. Antonio Esteve $n^{\circ} 20$. Barcelona: Fundación Dr. Antoni Esteve. pp85-102. [Consulta: 01 Abril 2016]

GUIFORD W.H. (2001). “Teaching Peer Review and The Process of Scientific Writing” Advances in Physiology Education, Vol. 25 (3), pp 167-175.

HERMOSILlA, Z., CLEMENTE, M., TRINIDAD, Á., \& ANDRÉS, J. (2013) “Competencia en comunicación oral: un reto para el ingeniero." En Garrigos et al. "New changes in technology and innovation.” INNODOCT/13. Universidad Politécnica de Valencia.

MÁRQUEZ, N. P. (2011). Modelo de evaluación de la comunicación oral en el proceso de enseñanza/aprendizaje de la interpretación simultánea. redit: Revista electrónica de didáctica de la traducción y la interpretación, (7), 1-15.

MOON SOOK Y.; SUN-MI C. (2011). "Effects of Peer Review on Communication Skills and Learning Motivation Among Nursing Students” Journal of Nursing Education, Vol. 50 (4), pp 230233. < http://dx.doi.org/10.3928/01484834-20110131-03> [Consulta: 29 de Marzo de 2016].

RIEMER Marc J. (2007). “Communication Skills for the 21st Century Engineer” Global J. of Engng. Educ., Vol. 11 (1), pp 89-100.

SOLANO, J.P., DE LA FUENTE, M.V., CONESA, H.M., AZNAR, A. (2011). Desarrollo y evaluación de la competencia Comunicación oral en titulaciones técnica: Estudio de Casos. Congreso internacional de innovación docente. Disponible en: http://repositorio.bib.upct.es/dspace/bitstream/10317/2280/1/c214.pdf (12/03/2012).

STEPHANIE N. (2000). “Teaching Collaborative Writting and Peer Review Techniques to Engineering and Technology Undergraduates” en Frontiers in Education Conference. Kansas City, MO. Disponible en < http://dx.doi.org/10.1109/FIE.2000.896636> [Consulta: 29 de marzo de 2016]. 
ANEXO I.

\section{Trabajos propuestos}

1. Sistema respiratorio

1.1 Estructura anatómica

1.2 Unidades funcionales

1.3 Funciones de la respiración

1.4 Sistemas de registro de la respiración

2. Sistema Nervioso

2.1 Estructura anatómica

2.2 Funciones

2.3 Unidades básicas: glía y neurona

2.4 La sinapsis

2.5 Estímulo sensorial/impulso nervioso

2.6 Señal bioeléctrica cerebral, el EEG.

2.6.1 Sistemas y estándares de captación

2.6.2 Tipos de EEG

2.6.3 Ondas cerebrales

2.7 Alteraciones de la actividad bioeléctrica cerebral Parkinson y Epilepsia

3. Actividad muscular

3.1 Tipos de músculos. Estructura y composición

3.2 Músculos esqueléticos.

3.2.1 Electromiografía, características del EMG

3.2.2 Aplicaciones de la electromiografía: detección de la fatiga muscular

3.2.3 Otras aplicaciones de la electromiografía

4. Aparato digestivo

3.1 Estructura anatómica

3.2 Funciones

3.3 Señales bioeléctricas:
3.3.1 El electrogastrograma
3.3.2 El electroenterograma
3.3.4 El colonograma

5. Gestación

5.1 Fases fisiológicas

5.2 Anatomía

4.1 Señal mioeléctrica uterina, el electrohisterograma (EHG)

4.2 Aplicabilidad diagnóstica del EHG

6. Sistemas de ayuda al diagnóstico del Parkinson 6.1 Qué es el Parkinson.

(c) ) EY-Nc-No 2016, Universitat Politècnica de València

Congreso In-Red (2016) 
Análisis del uso del póster científico y de la revisión por pares como herramienta desarrollo de la competencia comunicación efectiva en estudiantes de grado en ingeniería

6.2 Sistemas de diagnóstico: Evaluación de la marcha

6.2.1 Técnicas de medición

6.2.1.1 Tipos de electrodos, colocación

6.2.2 Parametrización

(cc) EY-NC-ND 2016, Universitat Politècnica de València 
ANEXO II.

\section{Cuestionario Trabajo Pósteres Bioelectrónica Curso 15-16}

1. ¿Desde su punto de vista, en general los ponentes dominan la materia relativa al trabajo?

$\square$ Totalmente de acuerdo

$\square$ Más bien de acuerdo

$\square$ Más bien en desacuerdo

$\square$ Totalmente en desacuerdo

2. Indique el trabajo que le ha resultado más atractivo:

$\square 1 \quad \square 2 \square 3 \square 4 \quad \square 5 \square 6 \square 7$

3. Indique el trabajo que se explicó con mayor claridad:

$\square 1 \quad \square 2 \square 3 \square 4 \quad \square 5 \square 6 \quad \square 7$

4. ¿ ¿Cuántas horas de trabajo han invertido en la recopilación de la información para la elaboración de la primera versión del póster?

$\square<10 \mathrm{~h}$

$\square 10-20 \mathrm{~h}$

$\square>20 \mathrm{~h}$

5. ¿Cuántas horas han invertido en la elaboración del póster?

$$
\begin{aligned}
& \square<5 \mathrm{~h} \\
& \square 5-15 \mathrm{~h} \\
& \square>15 \mathrm{~h}
\end{aligned}
$$

6. ¿Cuántas horas han invertido en la revisión del póster de los compañeros?

$\square<1 \mathrm{~h}$

$\square 1-3 \mathrm{~h}$

$\square>3 \mathrm{~h}$

(c)) EY-Nc-No 2016, Universitat Politècnica de València

Congreso In-Red (2016) 
Análisis del uso del póster científico y de la revisión por pares como herramienta desarrollo de la competencia comunicación efectiva en estudiantes de grado en ingeniería

7. ¿Cuantas horas de trabajo requieren para la elaboración de la segunda versión del póster, así como la calidad del póster?

$\square<1 \mathrm{~h}$

$\square 1-5 \mathrm{~h}$

$\square>5 \mathrm{~h}$

8. ¿ ¿Consideran que la revisión por pares les permitido mejorar el contenido del trabajo a exponer?

$\square$ Totalmente de acuerdo

Más bien de acuerdo

$\square$ Más bien en desacuerdo

$\square$ Totalmente en desacuerdo

9. ¿Consideran que los comentarios y/o sugerencias de los revisores eran objetivos y les han ayudado a mejorar el contenido del trabajo a exponer y/o la calidad del póster?

$\square$ Totalmente de acuerdo

$\square$ Más bien de acuerdo

$\square$ Más bien en desacuerdo

$\square$ Totalmente en desacuerdo

10. ¿Consideran que la revisión por pares es una buena metodología docente para mejorar la comunicación visual del póster y por tanto deberán implantarse para los cursos posteriores?

$\square$ Totalmente de acuerdo

Más bien de acuerdo

$\square$ Más bien en desacuerdo

$\square$ Totalmente en desacuerdo

(cc) EY-Nc-No 2016, Universitat Politècnica de València 